\section{BRAZIULIAN JOURNAL}

OF MIEDICAL AND BIOLOGICAL RESFARCH

www.bjournal.com.br
ISSN 0100-879X

Volume 43 (01) 1-123 January 2010

BIOMEDICAL SCIENCES

AND

CLINICAL INVESTIGATION

Braz J Med Biol Res, J anuary 2010, Volume 43(1) 115-119

Cross-transmission of vancomycin-resistant Enterococcus in patients undergoing dialysis and kidney transplant

D. Fram, F.M. Castrucci, M. Taminato, P. Godoy-Martinez, M.C.S. Freitas, A. Belasco, R. Sesso,

A. Pacheco-Silva, A.C. Pignatari and D. Barbosa

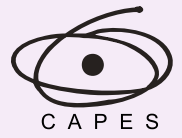

Ministério da Educação



Institutional Sponsors 


\title{
Cross-transmission of vancomycin-resistant Enterococcus in patients undergoing dialysis and kidney transplant
}

\author{
D. Fram¹, F.M. Castrucci², M. Taminato ${ }^{1}$, P. Godoy-Martinez ${ }^{2,4}$, M.C.S. Freitas ${ }^{3}$, \\ A. Belasco ${ }^{1}$, R. Sesso ${ }^{3}$, A. Pacheco-Silva ${ }^{3}$, A.C. Pignatari² and D. Barbosa ${ }^{1}$ \\ ${ }^{1}$ Departamento de Enfermagem, ${ }^{2}$ Departamento de Medicina, Disciplina de Infectologia, \\ ${ }^{3}$ Departamento de Medicina, Disciplina de Nefrologia, Universidade Federal de São Paulo, \\ São Paulo, SP, Brasil \\ ${ }^{4}$ Institute of Microbiology, Universidad Austral de Chile, Valdivia, Chile
}

\begin{abstract}
The objective of this study was to investigate the occurrence of vancomycin-resistant Enterococcus (VRE) cross-transmission between two patient groups (long-term dialysis and kidney transplant patients). Molecular typing, by automated ribotyping with the RiboPrinter Microbial Characterization System (Qualicon, USA), was used to analyze VRE isolates from 31 fecal samples of 320 dialysis patients and 38 fecal samples of 280 kidney transplant patients. Clonal spread of $E$. faecalis and $E$. casseliflavus was observed intragroup, but not between the two groups of patients. In turn, transmission of E. gallinarum and E. faecium between the groups was suggested by the finding of vancomycin-resistant isolates belonging to the same ribogroup in both dialysis and transplant patients. The fact that these patients were colonized by VRE from the same ribogroup in the same health care facility provides evidence for cross-transmission and supports the adoption of stringent infection control measures to prevent dissemination of these bacteria.
\end{abstract}

Key words: Vancomycin-resistant Enterococcus; Transmission; Dialysis; Kidney transplant

\section{Introduction}

Cross-transmission of multidrug-resistant organisms (MDRO), very often via hands of health care workers, has been a major factor accounting for the increase in MDRO incidence and prevalence, especially for methicillin-resistant Staphylococcus aureus and vancomycin-resistant enterococci (VRE) in acute care facilities (1). Among the many species of MDRO, enterococci have emerged as increasingly important nosocomial and community-acquired pathogens, since their tolerance against harsh conditions renders them difficult to control in health care environments $(2,3)$.

VRE infection has become a matter of great concern in patients with end-stage renal disease in America and Europe, and it has significantly contributed toward increasing the morbidity and mortality within this population (4). In 2003, VRE was identified in dialysis and renal transplant patients at the Universidade Federal de São Paulo nephrology service. The prevalence of colonization was $14.4 \%$ in dialysis patients, with type of dialysis treatment (hemodialysis vs peritoneal dialysis), hospital admission and length of hospital stay identified as risk factors. In kidney transplant patients, the observed prevalence was $13.6 \%$, and none of these risk factors were identified $(4,5)$. This observation strongly suggests the occurrence of nosocomial cross-transmission.

The aim of this study was to investigate the occurrence of VRE cross-transmission between patients undergoing longterm dialysis and kidney transplant patients at UNIFESP.

\section{Material and Methods}

This cross-sectional study was approved by the Research Ethics Committee at UNIFESP. All patients provided written informed consent for storage and later use of their biological materials.

Two to four samples of feces were collected in sterilized receptacles from each patient once a week during a

Correspondence: D. Barbosa, Departamento de Enfermagem, UNIFESP, Rua Diego Suarez, 45, 04928-190 São Paulo, SP, Brasil.

E-mail: dulce.barbosa@unifesp.br or dayana.fram@unifesp.br

Received June 16, 2009. Accepted November 16, 2009. Available online December 7, 2009. Published January 11, 2010. 
1-month period. Thirty-one VRE isolates were obtained from feces of 320 dialysis patients, and 38 VRE isolates from feces of 280 kidney transplant patients $(4,5)$. VRE samples were stored at $-20^{\circ} \mathrm{C}$ in glycerol media at the UNIFESP Special Clinical Microbiology Laboratory until retrieval for the present study.

Between December 2007 and July 2008, these VRE isolates were submitted to further characterization in azide blood agar supplemented with $6 \mu \mathrm{g} / \mathrm{mL}$ vancomycin (6). They were identified at the species level by conventional biochemical tests as described by Facklam et al. (7). Vancomycin resistance was confirmed by disk diffusion and the E-test (AB Biodisk, Sweden) according to Clinical and Laboratory Standards Institute (CLSI) breakpoints (8).

\section{Molecular typing}

Molecular typing was performed by automated ribotyping, employing the RiboPrinter Microbial Characterization System (Qualicon, USA). This automated process includes cell lysis, DNA cleavage by restriction enzymes (EcoRI), and band separation using electrophoresis gel and the modified Southern blot technique. DNA fragments were hybridized with a labeled universal probe derived from ribosomal RNA from Escherichia coli, and bands were detected by means of a luminescent chemical substrate. Images were captured on a camera and electronically transferred to a computer coupled to the ribotyping system. Every line representing sample data was normalized in accordance with a standard marker based on the intensity of the bands. The coefficient of similarity was calculated by the computing system on the basis of the position and relative weight of the bands. All samples with band patterns presenting a coefficient of similarity $\geq 0.90$ were included in the same ribogroup. The samples with coefficients of similarity below 0.90 were classified into distinct ribogroups (9).

\section{Dendrogram analysis}

The identified ribogroups were exported as a TIFF file and imported into the BioNumerics ${ }^{\mathrm{TM}}$ software (Applied Maths, Belgium). The patterns were normalized based on the mobility of standards, and a similarity matrix was created. The clustering was developed on the basis of the unweighted pair group method with arithmetic averages (UPGMA). The Dice correlation coefficient was used to analyze the similarities of the banding patterns. Based on the use of internal controls in the database, pattern optimization and band position tolerance of 0.8 and 1.0, respectively, were allowed.

\section{Results}

The following species were identified by automated ribotyping in $31 \mathrm{VRE}$ isolates from dialysis patients: $E$. faecalis $(\mathrm{N}=2)$, E. casseliflavus $(\mathrm{N}=5)$, E. faecium $(\mathrm{N}=9)$, and $E$. gallinarum $(\mathrm{N}=15)$. In the 38 samples from kidney transplant patients, E. faecalis $(\mathrm{N}=10)$, E. casseliflavus $(\mathrm{N}=10)$, E. faecium $(\mathrm{N}=10)$, and $E$. gallinarum $(\mathrm{N}=8)$ were observed.

Following automated ribotyping, computational analysis using the BioNumerics ${ }^{\mathrm{TM}}$ software was used to compare typing data for all bacterial isolates in order to group the organisms according to degree of similarity. This allows determination of clusters sharing a common source of infection. A similarity coefficient of $80 \%$ was selected to define ribogroup clusters after reviewing the epidemiologic data associated with each of the clusters of MDRO clones (10-12). Thirty-five ribogroups were found: $E$. faecalis with 3 ribogroups, E. casseliflavus with 14 ribogroups, E. faecium with 13 ribogroups, and $E$. gallinarum with 7 ribogroups.

E. faecium 112-S-4 was observed in 4 hemodialysis patients and in 1 transplant patient. As shown in Figure 1A, these samples had a coefficient of similarity $>83 \%$, suggesting clonal dissemination. The predominant ribogroup was E. gallinarum 112-S-4, which colonized 13 hemodialysis patients and four transplant patients, with a $92 \%$ coefficient of similarity, again suggesting clonal dissemination between the two patient groups. Furthermore, ribogroup 116-S-3 observed in one kidney transplant patient showed more than $84 \%$ similarity with the $112-$ S-4 ribogroup, indicating a possible relationship between these samples (data not shown).

The largest ribogroup diversity was observed for $E$. casseliflavus samples. Nevertheless, only one E. casseliflavus ribogroup, namely 112-S-4, was detected in two kidney transplant samples (intragroup transmission). It should be noted, however, that dendrogram analysis of the E. casseliflavus 112-S-4 ribogroup revealed a coefficient of similarity of $87 \%$ with the $115-\mathrm{S}-4$ ribogroup from hemodialysis patient samples (data not shown). Despite the fact that these are different ribogroups, this finding also supports the notion of cross-transmission between the two patient groups.

Of the E. faecalis samples from kidney transplant patients, nine were classified in the same ribogroup, 112-S-4. Only one sample was classified as belonging to a distinct ribogroup, 118-S-6. Interestingly, these ribogroups had a similarity $>87 \%$, suggesting cross-transmission of this pathogen within the transplant group. Based on dendrogram analysis, it is possible to confirm intragroup clonal spread of $E$. faecalis in hemodialysis patients, but not between the dialysis and transplant groups (Figure 1B).

\section{Discussion}

VRE infection is a growing problem in specific groups of patients. According to the National Healthcare Safety Network, $26 \%$ of blood cultures in outpatient dialysis are caused by VRE (13).

The resistance to glycopeptides might be mediated by various gene clusters: vanA, vanB, van $C$, vanD, vanE, vanG, and vanL (14). VanA-related clusters have high- 
A

E. faecium

Dice (Opt:0.80\%) (Tol 1.0\%-1.0\%) $(\mathrm{H}>0.0 \% \mathrm{~S}>0.0 \%)[0.0 \%-100.0 \%]$

Ribotyping

Ribotyping
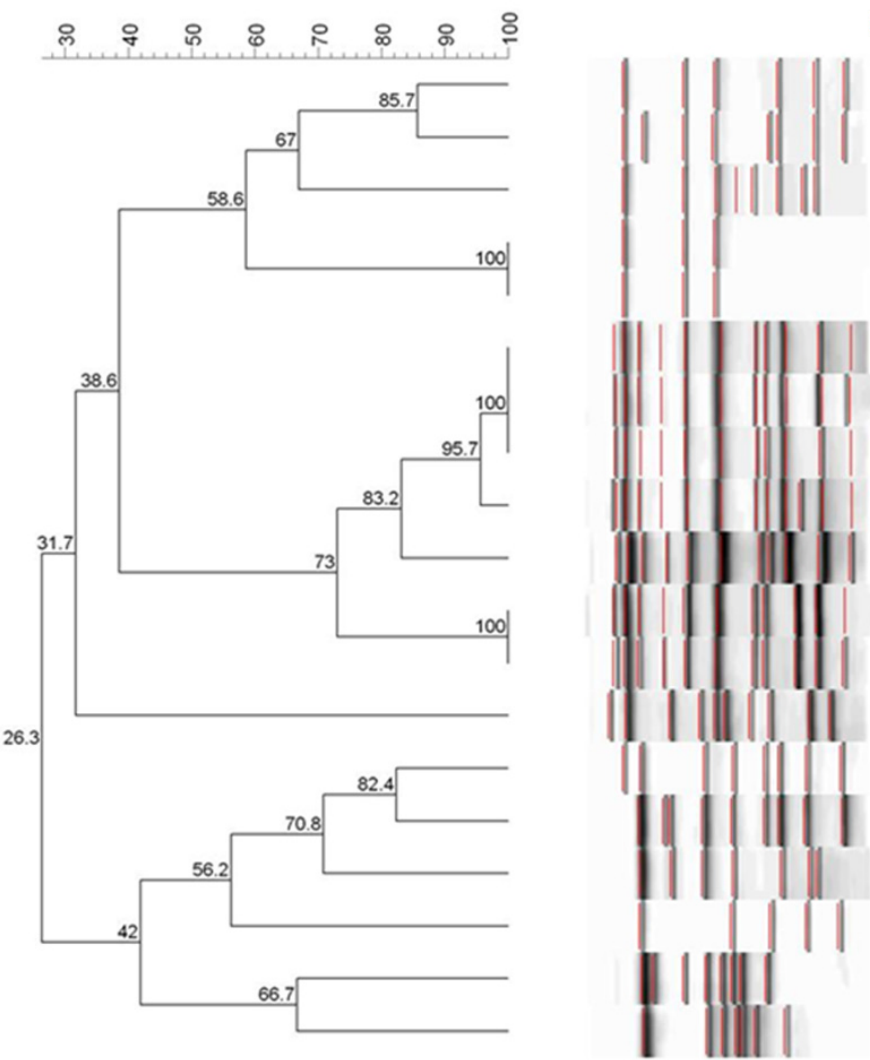

Sample Ribogroup Patient

A8422 111-S-4 KT

A8073 111-S-6 KT

A6178 111-S-5 KT

A8037 111-S-1 KT

A8084 111-S-1 KT

A5545 112-S-4 DL

A7581 112-S-4 KT

A7575 112-S-4 DL

A7656 112-S-4 DL

A6059 112-S-4 DL

A6229 113-S-6 DL

A6031 113-S-6 DL

A7658 113-S-4 DL

A8356 111-S-7 KT

A6712 17-S-6 KT

A5860 112-S-2 KT

A8357 111-S-8 KT

A7868 112-S-1 DL

A6448 113-S-1 DL

B

E. faecalis

Dice (Opt:0.80\%) (Tol 1.0\%-1.0\%) (H>0.0\% S>0.0\%) [0.0\%-100.0\%] Ribotyping

Ribotyping
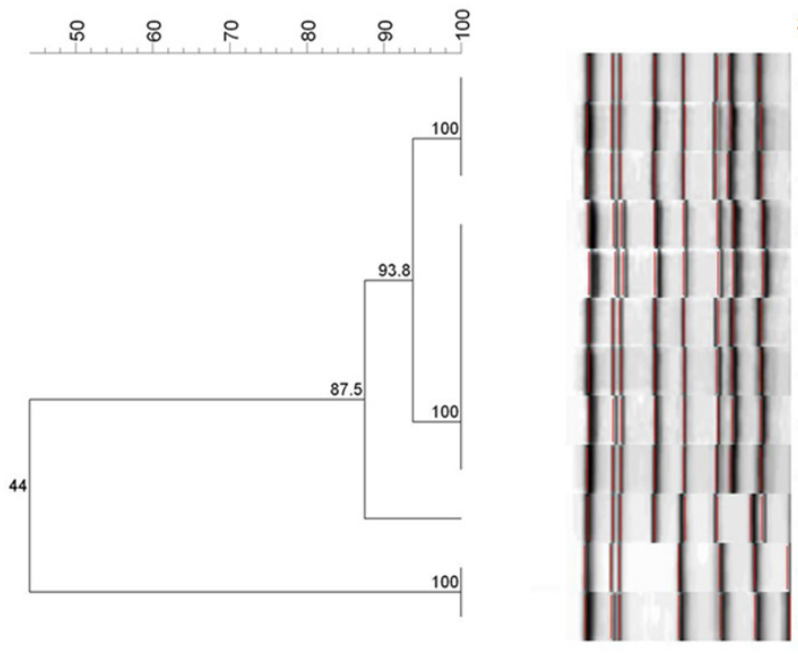

\begin{tabular}{|l|} 
Sample \\
A5292 \\
A5215 \\
A5232 \\
A5862 \\
A8391 \\
A5861 \\
A5544 \\
A5294 \\
A5291 \\
A8074 \\
A7573 \\
A6176 \\
\hline
\end{tabular}

Ribogroup
$118-S-4$
$118-S-4$
$118-S-4$
$118-S-4$
$118-S-4$
$118-S-4$
$118-S-4$
$118-S-4$
$118-S-4$
$118-S-6$
$16-S-8$
$16-S-8$

Patient

KT

KT

KT

KT

KT

KT

KT

KT

KT

KT

$\mathrm{DL}$

$\mathrm{DL}$

Figure 1. $A$, Dendrogram analysis of Enterococcus faecium ribogroups isolated from dialysis (DL) and kidney transplant patients (KT). $B$, Dendrogram analysis of $E$. faecalis ribogroups isolated from $\mathrm{DL}$ and $\mathrm{KT}$ patients. 
level resistance to vancomycin and teicoplanin (15). VanB isolates were believed to be inducibly resistant to lower levels of vancomycin; however, the range of resistance has been reported to be fairly broad ( 4 to $\geq 1000 \mu \mathrm{g} / \mathrm{mL}$ ) and susceptibility to teicoplanin is retained (16).

Gene resistance is mediated by mobile elements (plasmids) that can be transferred from one strain of Enterococcus to another. VanA-and vanB-resistant phenotypes have been described primarily in E. faecalis and E. faecium, which are responsible for most enterococcal infections and nosocomial VRE outbreaks, usually carried by gene vanA. E. gallinarum and E. casseliflavus are also occasionally responsible for infections (15).

In the present study, we showed that dialysis and kidney transplant patients were colonized with similar vancomycinresistant $E$. faecium and $E$. gallinarum ribogroups. VRE has become an important nosocomial pathogen because of its rapid spread, high mortality rates associated with infections, limited options for treatment and the possibility of transferring the vanA resistance gene to other more virulent and more prevalent pathogens, such as $S$. aureus (17).

McNeil et al. (18) showed that patients who acquired VRE after transplant had worse outcomes than those with VRE colonization documented before transplant (longer duration of hospitalization and intensive care unit stay and higher mortality rates). Mathematical modeling studies have been used to estimate the impact of active surveillance cultures to control MDRO. One such study evaluating interventions to decrease VRE transmission indicated that use of active surveillance cultures, versus no cultures, could potentially decrease transmission by $39 \%$, and that with pre-emptive isolation plus active surveillance cultures transmission could be decreased by $65 \%$ (19).

Our results show the VRE cross-transmission occurred between the two patient groups analyzed, kidney transplant and hemodialysis patients. Patient-to-patient transmission in health care settings has been a major factor accounting for the increase in VRE incidence and prevalence. In a study conducted by Padiglione et al. (20), the analysis of VRE strains isolated in an acute-care hospital suggested that most isolates were non-clonal on pulsed-field gel

\section{References}

1. CDC (Centers for Disease Control and Prevention). Guideline for isolation precautions: Preventing transmission of infectious agents in healthcare settings. Atlanta: CDC; 2007.

2. Cereda RF, Pignatari AC, Hashimoto A, Sader HS. In vitro antimicrobial activity against enterococci isolated in a university hospital in Brazil. Braz J Infect Dis 1997; 1: 83-90.

3. Mundy LM, Sahm DF, Gilmore M. Relationships between enterococcal virulence and antimicrobial resistance. Clin Microbiol Rev 2000; 13: 513-522.

4. Barbosa D, Lima L, Silbert S, Sader H, Cendoroglo M, Draibe $S$, et al. Evaluation of the prevalence and risk factors for electrophoresis (PFGE), and that the strict infection control procedures that were in place at each study site were reasonably effective in preventing the nosocomial transmission of dominant clones. Nevertheless, the fact that some strains had similar PFGE patterns suggests that hospital-related transmission of VRE cannot be ruled out.

In our patients, the finding of $E$. faecium and $E$. faecalis strains with the same or similar molecular profiles by ribotyping strongly suggests patient to patient transmission by a common source, justifying the implementation of strict barrier measures. Freitas et al. (5) observed an unexpectedly high rate of VRE colonization in kidney transplant patients, which is very similar to that observed in intensive care units. Furthermore, a high rate of VRE colonization was observed in outpatients, raising the question of whether surveillance should be extended to recently admitted patients. Due to the high costs involved in surveillance culture, this strategy could be reserved for patients with known risk factors, or for those with a higher probability of transmission.

Some measures must be established to prevent the emergence and transmission of VRE. These include environmental measures such as cleaning and disinfection of patient care areas and equipment, single-patient use of non-critical equipment, and decolonization therapy when appropriate. Other useful measures include adequate dimensioning of the nursing staff, communication systems, education and training of medical and other healthcare personnel, judicious antibiotic use, performance improvement processes to ensure adherence to recommended infection control procedures, and comprehensive surveillance for application of MDRO infection control precautions during patient care (19).

Cross-transmission of VRE was observed between two groups of patients at a university hospital. It is possible that the dissemination of MDRO reported in this study may have been caused by factors that can be modified so as to benefit patients, staff, and the institution.

\section{Acknowledgments}

Research supported by FAPESP. colonization by vancomycin-resistant Enterococcus among patients on dialysis. Am J Kidney Dis 2004; 44: 337-343.

5. Freitas MC, Pacheco-Silva A, Barbosa D, Silbert S, Sader H, Sesso R, et al. Prevalence of vancomycin-resistant Enterococcus fecal colonization among kidney transplant patients. BMC Infect Dis 2006; 6: 133.

6. Grayson ML, Grabsch EA, Johnson PD, Olden D, Aberline $\mathrm{M}$, Li HY, et al. Outcome of a screening program for vancomycin-resistant enterococci in a hospital in Victoria. Med $J$ Aust 1999; 171: 133-136.

7. Facklam R, Sham DA, Teixeira LM. Enterococcus. In: Murray 
PR, Baron EJ, Pfaller MA, Tenover FC, Yolken RH (Editors), Manual of clinical of microbiology. 7th edn. Washington: ASM; 1999. p 297-305.

8. CLSI (Clinical and Laboratory Standards Institute). Performance standards for antimicrobial susceptibility testing. 18th edn. USA: Suppl M100-S18; 2008.

9. Bruce J. Automated system rapidly identifies and characterizes microorganisms in food. Food Technol 1996; 50: 7781.

10. Turton JF, Kaufmann ME, Warner M, Coelho J, Dijkshoorn $\mathrm{L}$, van der Reijden $\mathrm{T}$, et al. A prevalent, multiresistant clone of Acinetobacter baumannii in Southeast England. J Hosp Infect 2004; 58: 170-179.

11. Grothues D, Tummler B. New approaches in genome analysis by pulsed-field gel electrophoresis: application to the analysis of Pseudomonas species. Mol Microbiol 1991; 5: 2763-2776.

12. McDougal LK, Steward CD, Killgore GE, Chaitram JM, McAllister SK, Tenover FC. Pulsed-field gel electrophoresis typing of oxacillin-resistant Staphylococcus aureus isolates from the United States: establishing a national database. $J$ Clin Microbiol 2003; 41: 5113-5120.

13. Klevens RM, Edwards JR, Andrus ML, Peterson KD, Dudeck MA, Horan TC. Dialysis Surveillance Report: National Healthcare Safety Network (NHSN)-data summary for 2006. Semin Dial 2008; 21: 24-28.
14. Werner G, Coque TM, Hammerum AM, Hope R, Hryniewicz W, Johnson A, et al. Emergence and spread of vancomycin resistance among enterococci in Europe. Euro Surveill 2008; 13: 1-11.

15. Arthur M, Courvalin P. Genetics and mechanisms of glycopeptide resistance in enterococci. Antimicrob Agents Chemother 1993; 37: 1563-1571.

16. Cetinkaya Y, Falk P, Mayhall CG. Vancomycin-resistant enterococci. Clin Microbiol Rev 2000; 13: 686-707.

17. Chang S, Sievert DM, Hageman JC, Boulton ML, Tenover FC, Downes FP, et al. Infection with vancomycin-resistant Staphylococcus aureus containing the vanA resistance gene. N Engl J Med 2003; 348: 1342-1347.

18. McNeil SA, Malani PN, Chenoweth CE, Fontana RJ, Magee JC, Punch JD, et al. Vancomycin-resistant enterococcal colonization and infection in liver transplant candidates and recipients: a prospective surveillance study. Clin Infect Dis 2006; 42: 195-203.

19. CDC (Centers for Disease Control and Prevention). Management of multidrug-resistant organisms in healthcare setting. Atlanta: CDC; 2006.

20. Padiglione AA, Wolfe R, Grabsch EA, Olden D, Pearson $S$, Franklin $C$, et al. Risk factors for new detection of vancomycin-resistant enterococci in acute-care hospitals that employ strict infection control procedures. Antimicrob Agents Chemother 2003; 47: 2492-2498. 